\title{
SISTEMA DE COMUNICACIÓN Y CONTROL BASADO EN EL PENSAMIENTO
}

\section{SYSTEM OF COMMUNICATION AND CONTROL BASED ON THE THOUGHT}

\author{
Avid Román G. \\ TELECOM ParisTech, \\ 46 rue Barrault, 75013 - Paris, Francia
}

\section{RESUMEN}

El presente trabajo esta orientado a brindar un medio de comunicación a las muchas personas que sufren de una pérdida severa de función motora como resultado de diferentes accidentes y/o enfermedades, para que puedan controlar e interactuar mejor con su entorno, para lo cual se ha implementado una interfaz cerebro-computador a través de la adquisición de señales electroencefalograficas por medio de electrodos, implementación de algoritmos para extraer características y ejecutar un método de clasificación de tal manera de interpretar dichas señales y ejecutar acciones correspondientes.

El primer objetivo es diseñar y construir un Sistema de Comunicación y Control Basado en el Pensamiento capaz de captar y medir señales ElectroEncefaloGráficas (EEG).

El segundo objetivo es implementar el sistema de adquisición de datos incluyendo un filtro digital en tiempo real que nos permita eliminar el ruido.

El tercer objetivo es analizar la variación de las señales electroencefalográficas frente a las diferentes tareas bajo estudio e implementar un algoritmo de extracción de características.

El cuarto objetivo es trabajar sobre la base de las características de las señales electroencefalográficas, implementar un sistema de clasificación que permita discriminar las 2 tareas bajo estudio y en base a ello efectuar las acciones correspondientes.

Palabras clave: $\mathrm{BCI}, \mathrm{ICC}$, Control, AAR

\section{ABSTRACT}

The present work is oriented to offer a communication via to the many people who undergo of a severe loss of function motorboat as a result of different accidents and/or diseases, so that they can control and interact better with his environment, for which an brain-computer interface has been implemented through the acquisition of EEG signals by electrodes, implementation of algorithms to extract characteristics and to execute a method of classification of such way to interpret these signals and to execute corresponding actions.

The first objective to design and to construct to a System of Communication and Control Based on the Thought able to catch and to measures EEG signals.

The second objective is to implement the system of data acquisition including a digital filter in real time that allows us to eliminate the noise.

The third objective is to analyze the variation of the EEG signals in front of the different tasks under study and of implementing an algorithm of extraction of characteristics.

The fourth objective is to work on the base of the characteristics of the EEG signals, to implement a classification system that allows to discriminate the 2 tasks under study and on the basis of it of conducting the corresponding battles.

Keywords: BCI, ICC, Control, AAR 


\section{INTRODUCCION}

Hay un número significativo de personas que tienen discapacidades motoras severas debido a diversas causas, lesiones cervicales altas, a parálisis cerebrales, esclerosis múltiple o distrofias musculares, en estos casos los sistemas de comunicación basados en electroencefalograma (EEG) proveen una nueva forma de comunicación, ya sea para incrementar la integración en la sociedad o para proveer de un medio de control sin asistencia permanente en el entorno en el que se desenvuelven [6].

Existen diversas técnicas y paradigmas en la implementación de interfaces CerebroComputadora $(\mathrm{BCl})$. En general, las $\mathrm{BCl}$ se basan en detectar señales de EEG asociadas a ciertos estados mentales.

Una interfaz cerebro computadora es un sistema de comunicación que permite generar una señal de control a partir señales cerebrales, tales como el electroencefalograma y los potenciales evocados. La comunicación entre las dos partes esenciales que conforman la $\mathrm{BCl}$ (cerebro y computadora), está regido por el hecho de que el cerebro genera el comando y la computadora debe interpretarlo.

El desarrollo de $\mathrm{BCl}$ es una línea de investigación reciente, aunque ya se obtuvieron resultados alentadores que hacen pensar en la posibilidad de desarrollar una $\mathrm{BCl}$ con un desempeño de alrededor de $70 \%$ de eficacia.

\section{MARCO TEORICO}

EI Electroencefalograma (EEG).- Es un estudio de la función cerebral que recoge la actividad eléctrica del cerebro, Para recoger la señal eléctrica cerebral se utilizan electrodos colocados en el cuero cabelludo, a los que se añade una pasta conductora para posibilitar que la señal eléctrica cerebral, que es de una magnitud de microvoltios, se pueda registrar y analizar.

Las señales electroencefalográficas tienen diferentes ritmos dentro de la banda de frecuencia con las siguientes características [7] y [9].

Ritmo Alfa o Mu: Es una característico del estado de vigilia y de reposo físico y mental con los ojos cerrados.

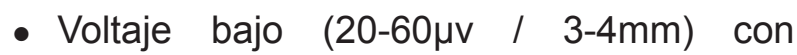
morfología variable.

- Frecuencia alta $(8-13 \mathrm{~Hz})$.

- Zonas de origen: posteriores.

- Bloqueo ante abertura palpebral y estímulos visuales (reactividad).

- Infancia no diferenciable, después de los 8 años $10 \mathrm{~Hz}$, plenamente establecido después de los 12 años.

Ritmo Beta: Es característico del estado de vigilia en estados de activación cortical (reemplazamiento de $\alpha$ ).

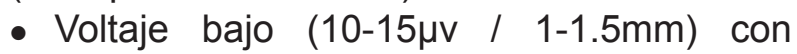
morfología variable.

- Frecuencia alta (13-25 ó $+\mathrm{Hz}$ ) a mayor frecuencia predominante en sujetos ansiosos, inquietos e inestables.

- Zonas de origen: frontales centrales.

Ritmo Tetha: Es característico del estado de sueño profundo y normal en la infancia (10 años), anormal durante la vigilia.

- Preponderante antes de los 2 años (situaciones emocionales).

- Aparición en condiciones fisiológicas específicas (hiperventilación y estados de sueño profundo).

- Voltaje alto $(50 \mu \mathrm{v} / 7 \mathrm{~mm})$.

- Frecuencia baja $(4-8 \mathrm{~Hz})$.

- Zonas de origen: zonas talamicas, localización parietotemporal.

Ritmo Delta: Es Característico de estados patológicos indicativos de sufrimiento neuronal (coma) y ocasional durante estados de sueño profundo.

- Voltaje alto $(70-100 \mu v$ / $9-14 \mathrm{~mm})$ con morfología variable.

- Frecuencia baja (4 ó - Hz).

- Origen subcortical (no definido).

Sistema internacional de Posición de Electrodos 10/20.- El electrodo inactivo o común se coloca alejado del cráneo (lóbulo de la oreja, nariz o mentón).

Se cuenta con puntos de referencia tales como: nasion e inion.

Diez por ciento por encima de los puntos de referencia se encuentran los planos pre frontal y occipital.

El resto esta dividido en cuatro partes iguales de $20 \%$ cada una. 
Existen cinco planos transversales:

- Prefrontal: Fpz

- Frontal: Fz

- Rolandico o vertex: $\mathrm{Cz}$

- Parietal: Pz

- Occipital: Oz

Plano horizontal: 10-20\% hacia la derecha e izquierda.
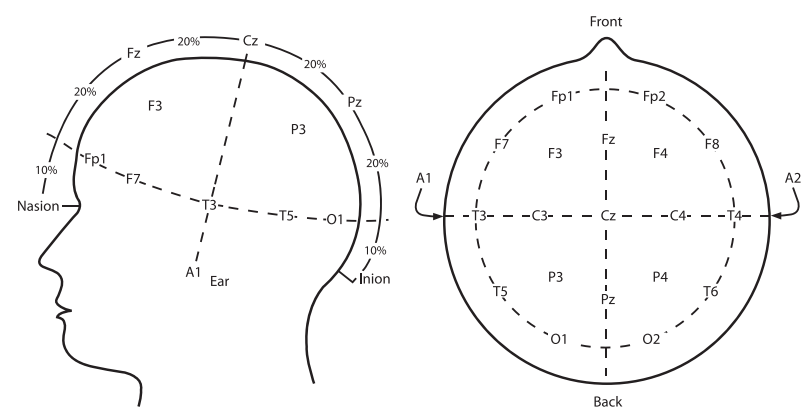

Figura 1. Posicionamiento de los Electrodos

\section{DESARROLLO DEL TRABAJO}

\section{Diagrama de Bloques}

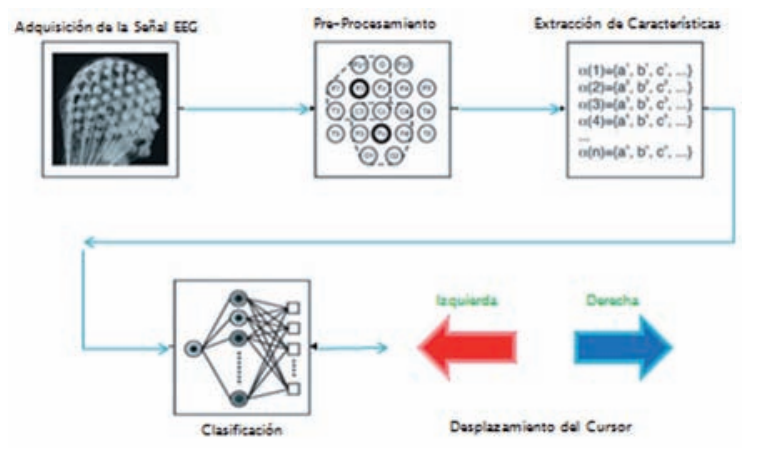

Figura 2. Diagrama de bloques.

Adquisición de Datos.- Para la adquisición de datos se utilizó electrodos de $8 \mathrm{~mm}$ de $\mathrm{Ag} / \mathrm{AgCl}$ fijados sobre la posición C3 y C4 del sistema internacional de posicionamiento 10/20. La señal se ha amplificado con un amplificador EEG de 8 canales modelo Procomp Infinity. La frecuencia de muestreo es de $256 \mathrm{~Hz}$.

Se ha implementado un filtro para banda digital entre 0.5 y $30 \mathrm{~Hz}$ en tiempo real para eliminar el ruido proveniente de distintas fuentes y en especial de la red eléctrica.

Figura 3. Electrodos

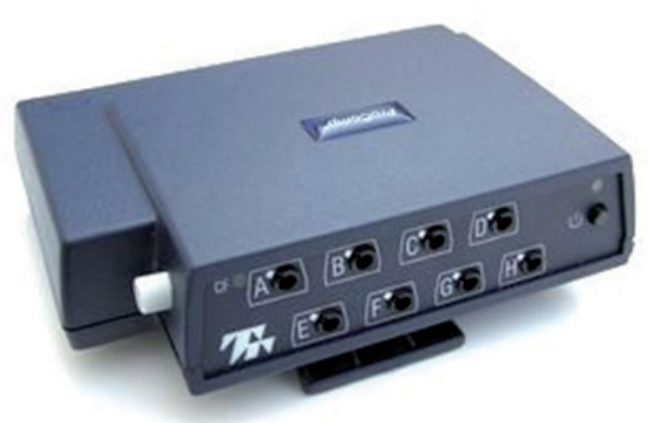

Figura 4. Amplificador Procomp Infinity

Extracción de Características.- La etapa de extracción de características es probablemente el paso más critico en el procesado de señal EEG. El objetivo de este paso es crear una representación manejable y significativa de la señal original EEG, con vistas a maximizar el éxito potencial de la etapa de clasificación y a su vez el rendimiento global del sistema. Un segundo objetivo de la etapa de extracción de características es comprimir los datos sin pérdida de información relevante, con objeto de reducir el número de variables de entrada en la fase de clasificación para que pueda operar en tiempo real.

El ritmo $\mu$, que corresponde a una oscilación de la señal EEG comprendida entre los 8 y $13 \mathrm{~Hz}$, es captado en la zona sensomotora situada en la región central del cuero cabelludo.

Dicho ritmo, presente en la mayoría de los adultos, tiene la particularidad de presentar una atenuación en su amplitud cuando se lleva a cabo algún tipo de movimiento, o lo que es más importante cuando se tiene la intención de realizar algún movimiento, o sencillamente imaginando movimientos de las extremidades, tal y como se muestra en las figuras $5,6,7$ y 8 [8].

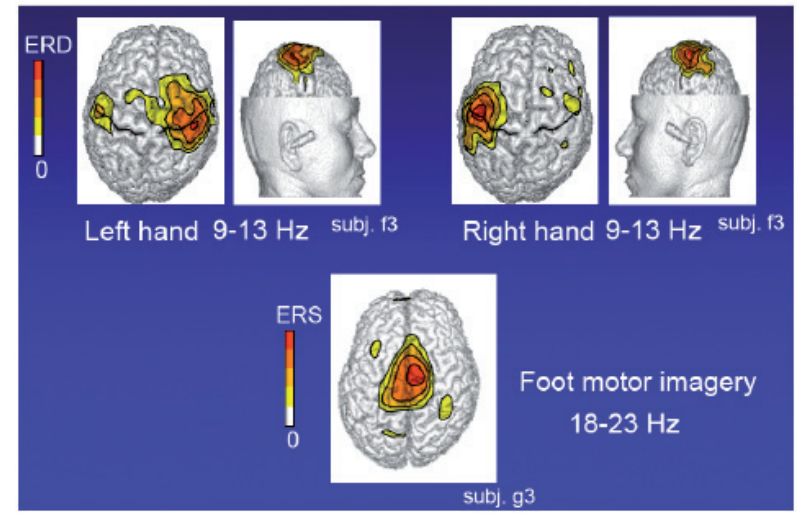

Figura 5. Actividad cerebral durante la imaginación de movimientos de la mano derecha e izquierda. 


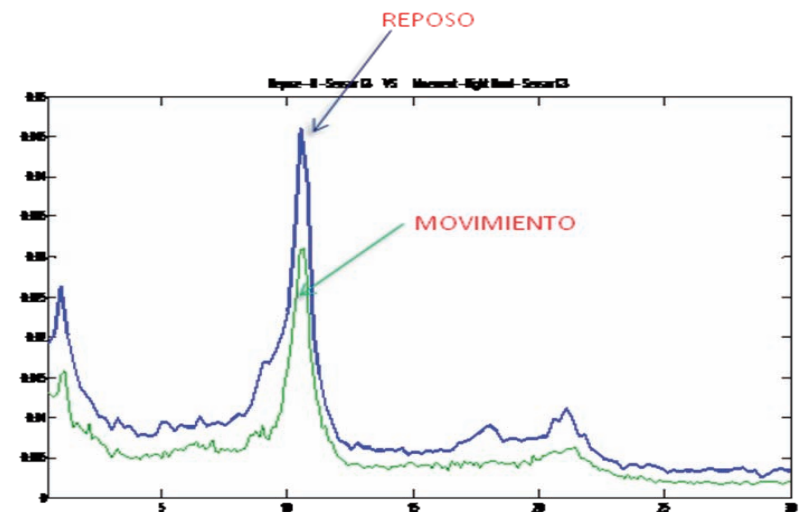

Figura 6. Diferencia en la banda de frecuencia alfa entre movimiento y reposo

Cabe recalcar que los movimientos de la mano derecha producen una variación en la actividad de la parte izquierda del cerebro y viceversa.

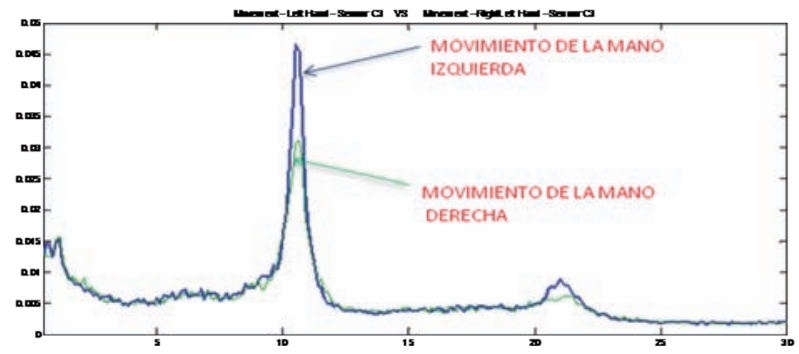

Figura 7. Diferencia en la banda de frecuencia alfa entre movimiento de la mano derecha e izquierda en el electrodo de la posición C3

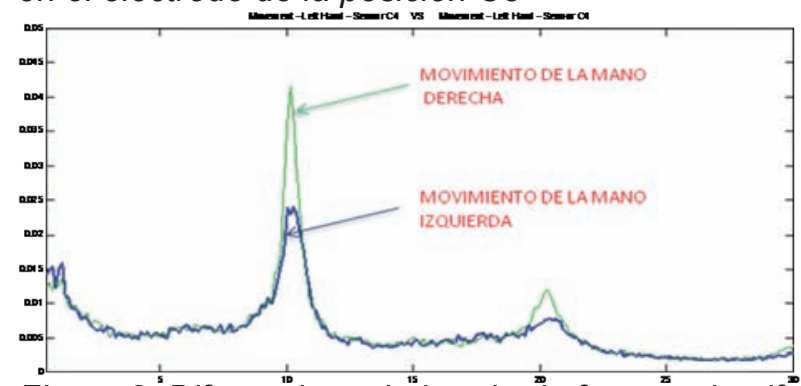

Figura 8.'Diferencia en la banda de frecuència alfäa entre movimiento de la mano derecha e izquierda en el electrodo de la posición C4

\section{Parámetros Adaptativos Auto Regresivos} (AAR).- [3][4]Para representar las características anteriormente descritas en cifras que nos permitan implementar un algoritmo de clasificación, utilizamos los parámetros adaptativos auto regresivos que nos permiten representar la respuesta en frecuencia de la señal tal y como se muestra en la figura 9.

Un modelo AAR de orden $p$ es escrita de la siguiente manera:

$$
\begin{aligned}
y(t) & =a_{1}(t) * y(t-1)+\ldots+a_{p}(t) * y(t-p)+x(t) \\
& =a(t)^{\top} * Y(t-1)+x(t)
\end{aligned}
$$

La diferencia con el moldeo estacionario auto regresivo $(A R)$ es que los parámetros $A A R$ varían con el tiempo; como consecuencia, la predicción del error se calcula de la siguiente manera:

$$
e(t)=y(t)-\hat{a}(t-1)^{\top} * Y(t-1)
$$

Para el cálculo de los parámetros existen bastantes métodos, en esta oportunidad utilizamos el método de Last-Mean-Squares (LMS) cuya formula esta dada por:

$$
\hat{a}(\mathrm{t})=\hat{a}(\mathrm{t}-1)+(0 \quad / M S Y) * e(t) * Y(t-1)
$$

Donde:

C $\rightarrow$ Coeficiente e Actualizacion $=0.0055$

MSY $\rightarrow$ Varianza e b Señal $=\frac{1}{N} \sum_{t-1}^{N} Y_{t}^{2}$
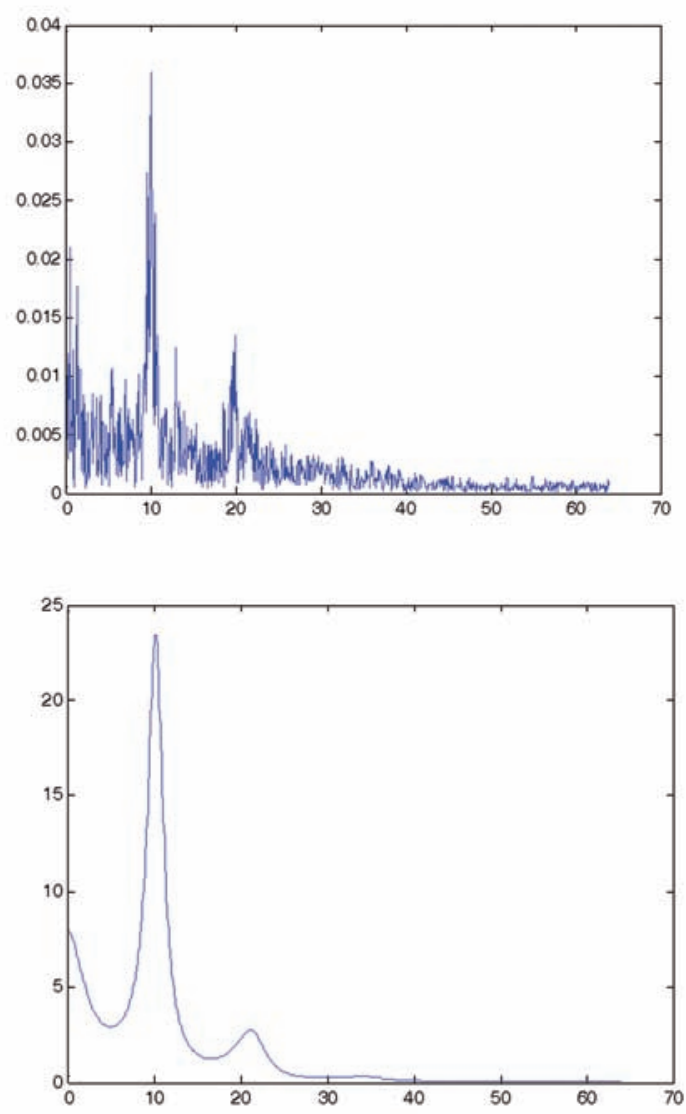

Figura 9. Comparación de Respuesta en Frecuencia con la FFT y los Parámetros AAR

En el presente trabajo se utilizaron 6 parámetros AAR por cada electrodo, es decir un conjunto de 12 características.

Clasificación.- La fase de clasificación es la tarea final del procesado. La entrada al algoritmo de clasificación es el conjunto de características extraídas en la etapa anterior y la salida es una indicación del estado mental 
del usuario. En este caso estamos trabajando con dos estados: Izquierda y Derecha.

Para el presente trabajo se ha desarrollado dos métodos de clasificación: Análisis Discriminante Lineal y Redes Neuronales, ambos métodos dan como resultado un vector de pesos mas una constante, de esta manera la función de activación vendría dada por:

$$
\begin{aligned}
& \mathbf{A C}=\sum \mathbf{x}_{\mathbf{i}}{ }^{*} \mathbf{w}_{\mathbf{i}}+\text { cte } \\
& \mathbf{A C}=\mathbf{X} * \mathbf{W}+\text { cte }
\end{aligned}
$$

Protocolo de Pruebas

Fijación de los Electrodos.- Se utilizan electrodos bipolares por lo que cada electrodo se coloca a 2.5 centímetros adelante y atrás de las posiciones $\mathrm{C} 3$ y $\mathrm{C} 4$ tal como los muestra las figuras 10 y 11.

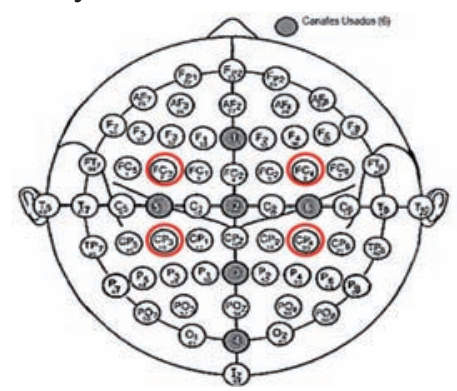

Figura 10. Fijación de los Electrodos Bipolares en las Posiciones C3 y C4

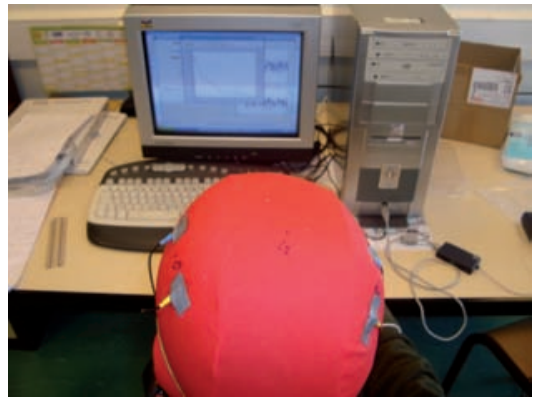

Figura 11. Fotografía con los Electrodos Fijados

Para fijar los electrodos se utiliza gel y pasta conductora.
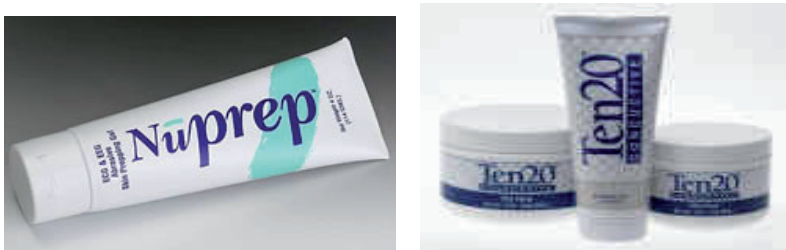

Figura 12. Gel y Pasta Conductora

Adquisición de la Señal y Entrenamiento.Cada uno de las pruebas dura solamente 9 segundos y durante el proceso de entrenamiento podemos tener 80 pruebas.
La prueba se inicia en reposo y a los 3 segundos el sistema escoge un valor aleatoriamente para mandar la señal ya sea de derecha o izquierda por lo que la persona tendrá los 6 segundos restantes para imaginar el movimiento especificado, para comprender mejor podemos observar la figura 13.

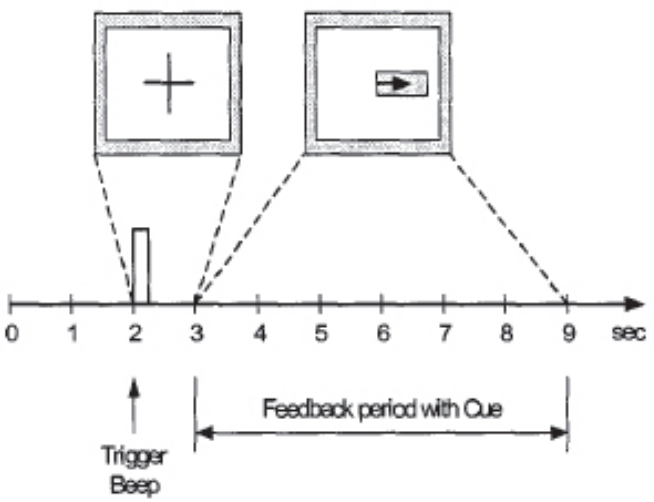

Figura 13. Composición de los 9 segundos de prueba [2]

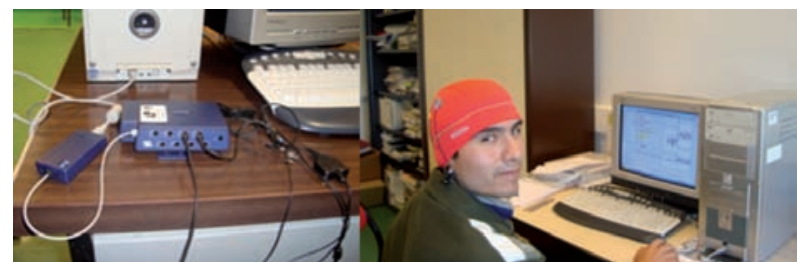

Figura 14. Fotografías durante el proceso de adquisición de las señales EEG

Para el entrenamiento se realizaron 2 etapas, la primera un entrenamiento OFFLINE en la cual no hay retroalimentación y sirve para registrar y guardar los datos para luego analizarlos de manera offline; la segunda es un entrenamiento ONLINE donde existe retroalimentación en función a los resultados preliminares del análisis offline, este entrenamiento online sirve para que el usuario pueda aprender a controlar de mejor manera la actividad cerebral. En las figuras 15 y 16 podemos observar los formularios implementados para cada uno de los entrenamientos.

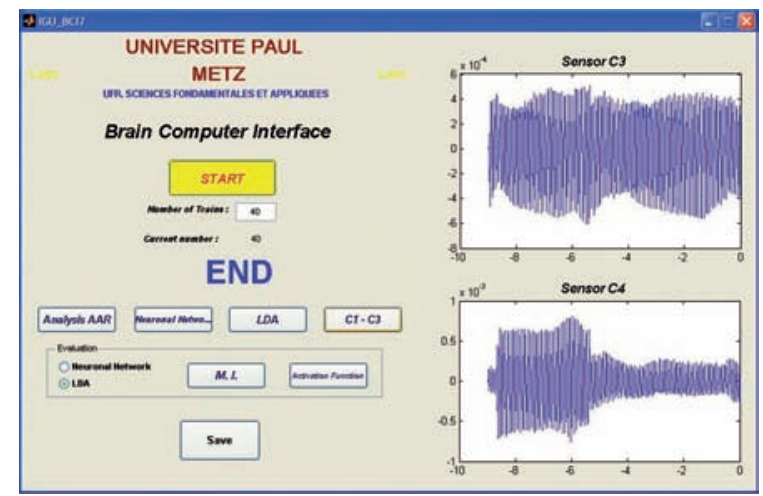

Figura 15. Interface para el Entrenamiento OFFLINE 


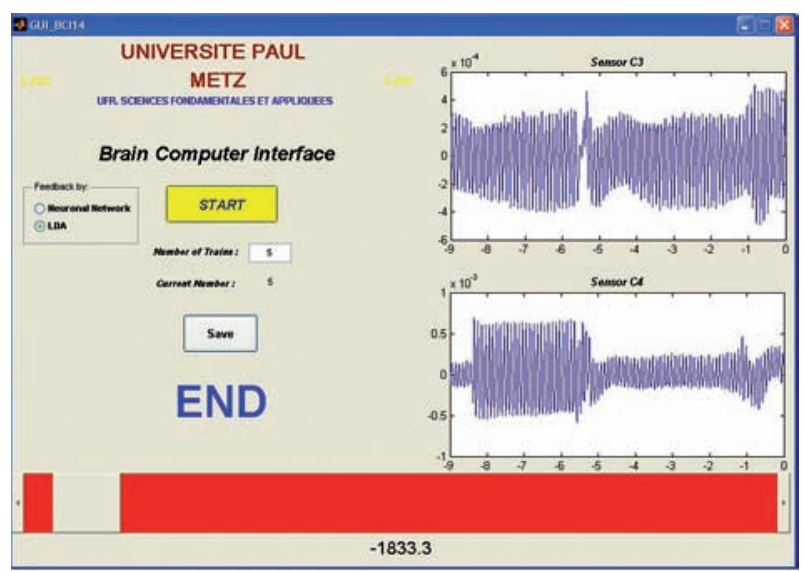

Figura 16. Interface para el Entrenamiento ONLINE con retroalimentación

\section{RESULTADOS}

Para poder evaluar los resultados obtenidos se tomó en cuenta dos métodos:

Error Rate.- Es el error que se produce al tratar de clasificar las señales producidas entre los dos tipos de tareas bajo estudio (movimiento de la mano derecha y movimiento de la mano izquierda).

Mutual Information.- es la cantidad de información que se puede recuperar a través de la clasificación y las características extraídas [1].

Como el análisis se hizo de manera continua pues la evaluación se realizó en cada instante de tiempo con cada muestra de dato adquirido por lo que en las figuras 17, 18, 19 y 20 podemos observar los valores del error y de Mutual Information en función del tiempo.
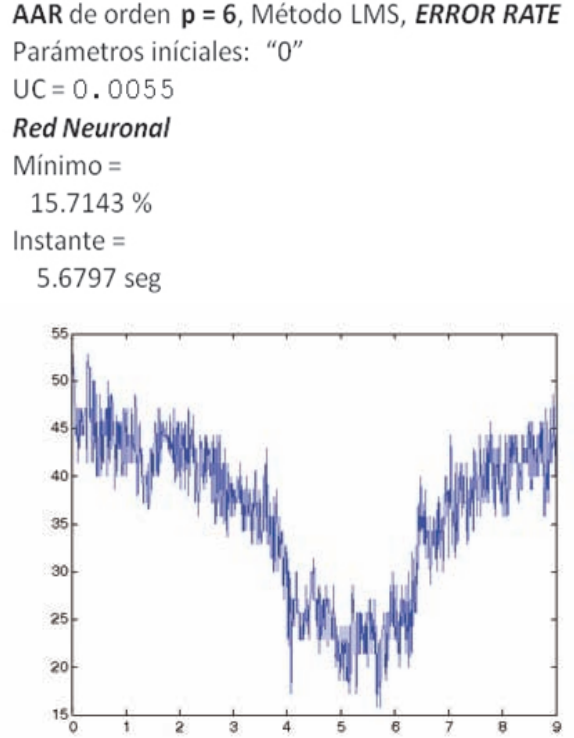

Figura 17. Grafica del Error con Redes Neuronales en Función del Tiempo
AAR de orden $\mathrm{p}=6$, Método LMS, ERROR RATE

Parámetros iníciales: " 0 "

$\mathrm{UC}=0.0055$

LDA

Mínimo $=$

$12.8571 \%$

Instante $=$

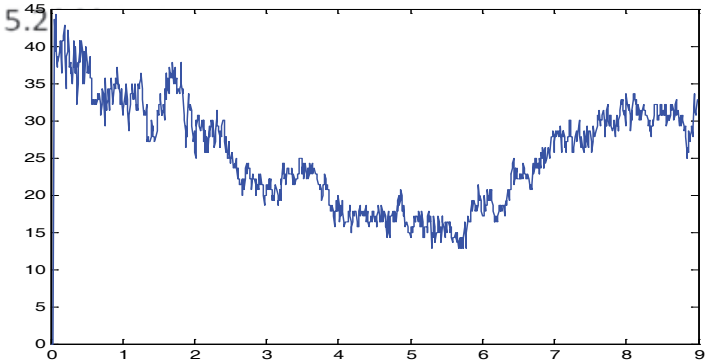

Figura 18. Gráfica del Error con LDA en función del tiempo

AAR de orden $p=6$, Método LMS, $M I$

Parámetros iníciales: " 0 "

$\mathrm{UC}=0.0055$

Red Neuronal

Máximo $=$

0.4583

Instante $=$

$5.7422 \mathrm{seg}$

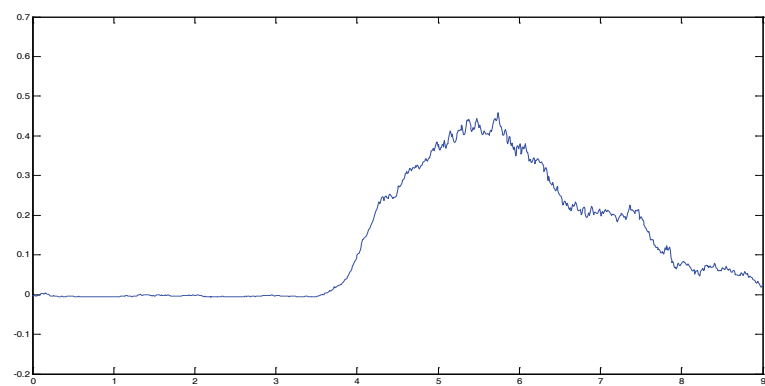

Figura 19. Gráfica Mutual Information con Redes Neuronales en Función del Tiempo

AAR de orden $p=6$, Método LMS, $M I$

Parámetros iníciales: " 0 "

$U C=0.0055$

LDA

Máximo =

0.5328

Instante =

$5.1563 \mathrm{seg}$

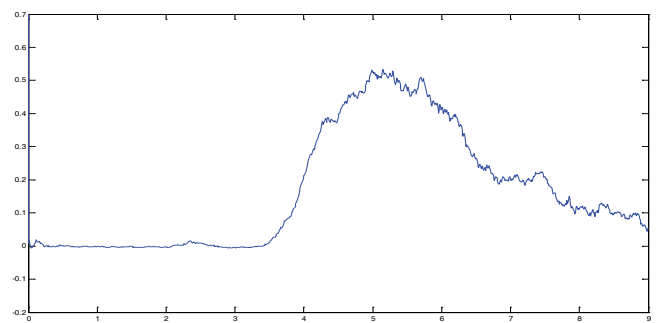

Figura 20. Grafica Mutual Information con LDA en función del tiempo. 
Se puede graficar la diferencia entre la energía espectral de la banda alfa $(8-13 \mathrm{~Hz})$ de $\mathrm{C} 3$ y C4 cuando se producen los dos tipos de movimiento. Así mismo podemos graficar la función de activación resultante del método de clasificación que se obtiene cuando el movimiento es hacia izquierda o derecha. Ambos gráficos están en función del tiempo debido al análisis continuo que se realizó en cada instante de tiempo, tal y como se muestra en las figuras 21 y 22 .

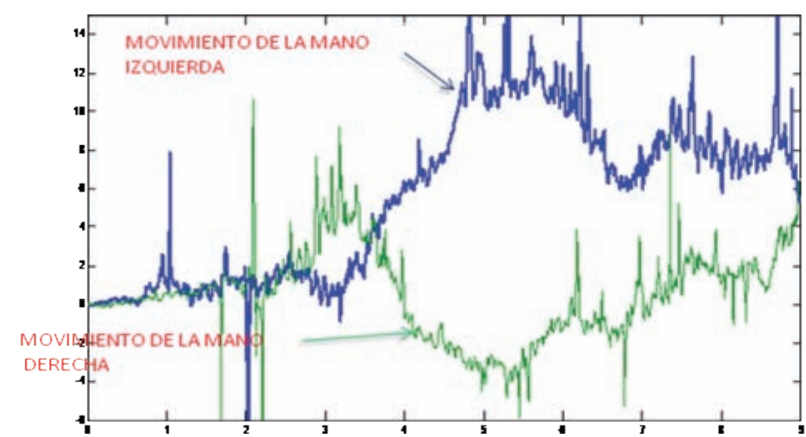

Figura 21. Diferencia de Energía Espectral entre los electrodos C3 y C4.

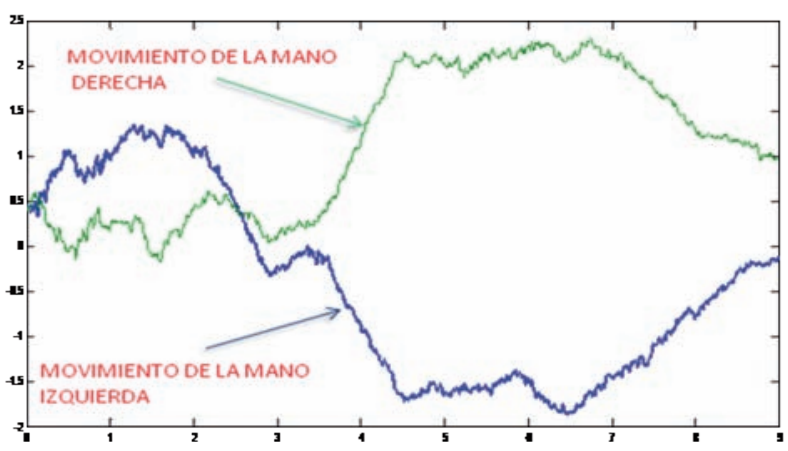

Figura 22. Gráfica de la Función de la Activación para movimientos de la mano derecha e izquierda

Mediante estas graficas podemos observar que las clases bajo estudio son separables.

\section{CONCLUSIONES}

Se logró la construcción de un sistema de comunicación y control basado en el pensamiento capaz de mover un cursor sobre la pantalla de un computador a partir de la actividad cerebral al momento de imaginar movimientos de la mano derecha e izquierda. La fijación de los electrodos debe realizarse de la mejor manera posible para poder medir una buena señal y no cometer errores, para lo cual se recomienda siempre hacer una verificación de la impedancia de los electrodos.

Cada individuo es distinto y la manera de controlar su actividad cerebral también es distinta, es por ello que para cada usuario diferente siempre es necesario realizar un entrenamiento previo.

Para poder obtener un buen entrenamiento es necesario realizar por lo menos 60 pruebas por cada individuo o usuario.

El análisis ha sido realizado de manera continua durante los 9 segundos de cada prueba y los mejores resultados tanto del mínimo error como el máximo valor de mutual information se encuentran entre el quinto y sexto segundo. Los errores encontrados con los métodos de clasificación LDA y Redes Neuronales son de $12.86 \%$ y $15.71 \%$ respectivamente.

Los valores para Mutual Information encontrados con los métodos de clasificación LDA y redes neuronales son de 0.5328 et 0.4583 respectivamente.

Los diferentes valores encontrados para el error pueden parecer altos, pero son valores comparables con los resultados obtenidos por otros grupos de investigación. 


\section{REFERENCIAS}

[1] A. Schlogl, C. Neuper, G. Pfurtscheller, "Estimating the Mutual Information of an EEG-based Brain-Computer Interface", Biomedizinische Technik 47(1 2): pp. 3-8, 2002.

[2] A. Schlogl, C. Keinrath, R. Scherer, G. Pfurtscheller, "Information transfer of an EEG-based brain computer interface", Proc. International IEEE EMBS, pp. 641-644, 2003.

[3] A. Schlogl, S. J. Roberts, G. Pfurtscheller, "A criterion for adaptative autoregressive models", Proc. International IEEE EMBS, pp. 1581-1582, 2000.

[4] A. Schlogl, D. Flotzinger, G. Pfurtscheller, "Adaptative Autoregressive Modeling used for Single-trial EEG Classification", Biomedizinische Technik 42(1997): pp. 162-167.

[5] Vickneswaran Jeybalan, Andrews samraj, LooChu Kiong, "Motor Imaginary Signal Classification using Adaptive Recursive Bandpass Filter and Adaptive Autoregressive Models for Brain Machine Interface Designs", International Journal of Biological and Medical Sciences, 2008.
[6] Ulrich Hoffmann, Jean-Marc Vesin, Touradj Ebrahimi; "Recent Advances in Brain-Computer Interfaces"; Ecole Polytechnique Federale de Lausanne (EPFL), Switzerland; 2008.

[7] Ricardo Ron Angevin, Carmen García Berdonés, Antonio Díaz Estrella; "Desarrollo de una interface Cerebro Computador"; Departamento de Tecnología Electrónica de la universidad de Málaga; 2001.

[8] Bin Lou, Bo Hong; "Bipolar Electrode Selection for a Motor Imagery Based Brain-Computer Interface"; IOP Publishing-Journal of Neural Engineering 5 (2008) 342-349.

[9] G. Pfurtscheller, F. H. Lopes da Silva; "Event-relatedEEG/MEGSynchronization and Desynchronization: Basic Principles"; Clinical Neurophysiology 110 (1999) 1842-1857.

\section{E-mail: a.roman@ieee.org}

